\title{
Precipitation Effects on Motor Vehicle Crashes Vary by Space, Time, and Environmental Conditions 0
}

\author{
J. D. TAMERIUS \\ Department of Geographical and Sustainability Sciences, The University of Iowa, Iowa City, Iowa \\ X. ZHOU \\ Department of Management Sciences, Tippie College of Business, The University of Iowa, Iowa City, Iowa \\ R. MANTILLA \\ Department of Civil and Environmental Engineering, The University of Iowa, Iowa City, Iowa \\ T. GREENFIELD-HUITT \\ Highways Division, Iowa Department of Transportation, Ames, Iowa
}

(Manuscript received 15 January 2016, in final form 20 May 2016)

\begin{abstract}
Numerous studies have shown that precipitation has a significant impact on motor vehicle crashes. Hourly weather radar data with a 4-km resolution and over 600000 crashes from 2002 to 2012 in Iowa are used to assess the effects of precipitation on motor vehicle crashes. Using a matched pairs analysis, this study finds that the relative accident risk (RAR) across the state during the study period was $1.69[1.66,1.71]$. However, RAR increased to as high as $3.7[3.6,4.0]$ and as low as $1.1[1.0,1.2]$ for frozen and liquid precipitation types, respectively. RAR also varied significantly by hour of the day, with RAR near 2 in the late afternoon and 1.3 during the early morning hours, suggesting an interaction effect between precipitation and traffic volume and/or density on crash risk. The study also shows that interstates and major highways tend to have higher RAR than smaller roads, and it was able to identify locations that are particularly sensitive to precipitation with regard to crashes. This study can be used to inform future studies on the effects of weather and climate change on crashes.
\end{abstract}

\section{Introduction}

Approximately $23 \%$ of crashes in the United States occur in adverse weather conditions (U.S. DOT 2011). Adverse weather conditions, such as rain, snow, ice, fog, wind, and temperature, are known to increase the frequency of motor vehicle crashes (Qin et al. 2007; Andrey et al. 2003; Abdel-Aty et al. 2011; Basagaña et al. 2015).

Supplemental information related to this paper is available at the Journals Online website: http://dx.doi.org/10.1175/ WCAS-D-16-0009.s1.

Corresponding author address: James D. Tamerius, Department of Geographical and Sustainability Sciences, The University of Iowa, 316 Jessup Hall, Iowa City, IA 52242.

E-mail: james-tamerius@uiowa.edu
Precipitation modulates the risk of crashes through reduced visibility and road friction (Andrey and Yagar 1993). A meta-analysis of previous studies suggests that rainfall and snowfall increase crash rates by $71 \%$ and $84 \%$, respectively, on average (Qiu and Nixon 2008). Precipitation rate has also been shown to have a positive relationship with crash rates (Hambly et al. 2013).

Although nearly all studies have identified a positive relationship between precipitation and crash risk, the effects vary significantly across studies and study regions. For example, studies in the United States have found an increase in crash risk of approximately 58\% and $73 \%$ for rainfall and snowfall, respectively, whereas studies in Canada have shown larger average increases in crash rates during rainfall and snowfall events of $73 \%$ and $85 \%$, respectively (Qiu and Nixon 2008). Previous studies have shown spatial differences in the effect of precipitation 
on crashes are due to spatial variations in weather patterns (Edwards 1999), traffic types (Jaroszweski et al. 2010), and roadway terrain (Jung et al. 2010). Other factors that may affect spatial variation in precipitation effects on crash risk are driver training, road design, speed limits, and variations in the data and methods used across studies.

A significant issue with past studies is the lack of consistent and representative weather data for different areas. This is because previous studies typically investigated precipitation effects on crashes employing weather station data. In general, weather stations are sparsely distributed and thus do not necessarily provide accurate information about the weather conditions at the specific location of each crash. Further, weather stations are typically clustered in populated areas, which can result in biases when analyzing the effects of precipitation across rural and urban regions. Recently, weather radar was employed to provide a more representative measure of precipitation during vehicle crashes (Jaroszweski and McNamara 2014). Employing weather radar allows for a more systematic and unbiased estimate of precipitation and its effects on motor vehicle crash risk.

In this study we determine the effect of precipitation on crash risk across Iowa using highly resolved precipitation data from weather radar. Iowa is an ideal region to study the effects of precipitation due to an extreme range of conditions that characterize the region, including severe spring and summer downpours and significant snow and ice events during the winter. We assess how precipitation affects crashes across time, space, and environmental conditions.

\section{Methods}

\section{a. Data and preprocessing}

The Iowa Department of Transportation (DOT)'s Motor Vehicle Division maintains a crash database that describes reported motor vehicle accidents in Iowa. Crash data were obtained from the University of Iowa Injury Prevention Research Center (IPRC) for the years 2002-12. The precise location of the crash (latitude, longitude) and the time stamp corresponding to the estimated time of crash were used in this analysis. All crash times were rounded to the nearest hour. Crash records with missing location (latitude, longitude) or time information were removed. Days corresponding with publicly observed holidays in Iowa were also removed. Over 600000 motor vehicle crashes during the years 2002-12 were included in the analysis.

Data on precipitation and air temperature were gathered for $2002-12$ for the state of Iowa. To characterize precipitation conditions we used the stage IV radar-rainfall product developed by the NWS (Lin and Mitchell 2005). The dataset contains hourly precipitation totals $(\mathrm{mm})$ and water equivalent (for snow) on a 4-km grid across all of Iowa. In addition to precipitation data, we also employed temperature data from the Road Weather Information System (RWIS) to evaluate the effects of temperature on crash rates. The RWIS consisted of 77 unique observing stations (1-min sampling intervals) located on major roads across Iowa. An hourly mean of surface and air temperatures was calculated for data for each RWIS station.

Hourly temperature data for the nearest (Euclidean distance) RWIS station were assigned to each grid cell. Based on the location information (latitude, longitude) of the motor vehicle crashes, we mapped each crash event to a grid cell in the 4-km weather radar grid. Accordingly, each grid cell was assigned hourly crash totals, radarderived precipitation accumulations, and temperature.

\section{b. Matched pairs analysis}

We used a matched pairs analysis following Andrey et al. (2003). Matched pairs account for possible confounding factors that could fluctuate across hour, day of the week, or season (e.g., traffic volume, driver behavior, and driver demographics) when assessing the effect of precipitation on crash rates. Let $\mathbf{M}$ be a set of matched pairs of size $N \times 2$ such that

$$
\mathbf{M}=\left[\mathbf{M}_{1}, \mathbf{M}_{2}\right]=\left[\begin{array}{cc}
m_{1,2} & m_{1,2} \\
\vdots & \vdots \\
m_{n, 1} & m_{n, 2}
\end{array}\right]
$$

Each matched pair consists of the number of crashes during an hour with precipitation, $\mathbf{M}_{i, 1}$, and an hour one week before or after with no precipitation, $\mathbf{M}_{i, 2}$. For example, if it rained on Friday at noon, it was matched with the previous or following Friday at noon assuming precipitation was absent. If precipitation was present during both the previous and following Friday at noon, then no matched pair was generated. We used the matched pairs to calculate the relative accident risk (RAR) by summing the number of crashes for hours with precipitation and dividing by the number of crashes with no precipitation across matched pairs:

$$
R=\frac{\sum_{j=1}^{n} m_{j, 1}}{\sum_{i=1}^{n} m_{i, 2}},
$$

where $R$ is the relative accident risk and $n$ is the number of matched pairs. The matched pairs analysis was conducted 
TABLE 1. List of variables, window spans, and intervals used in the stratified analyses.

\begin{tabular}{lll}
\hline \multicolumn{1}{c}{ Variable(s) } & Window span & \multicolumn{1}{c}{ Interval } \\
\hline Hour of day & $3 \mathrm{~h}$ & $1 \mathrm{~h}$ \\
Day of week & $1 \mathrm{day}$ & 1 day \\
Hour of week & $3 \mathrm{~h}$ & $1 \mathrm{~h}$ \\
Days of year & 15 days & 1 day \\
Temp & $1.5^{\circ} \mathrm{C}$ & $0.1^{\circ} \mathrm{C}$ \\
Lag & $1 \mathrm{~h}$ & $1 \mathrm{~h}$ \\
Precipitation rate & $3 \mathrm{~mm} \mathrm{~h}^{-1}$ & $0.1 \mathrm{~mm} \mathrm{~h}^{-1}$ \\
Precipitation rate and temp & $2 \mathrm{~mm} \mathrm{~h}^{-1}$ & $0.1 \mathrm{~mm} \mathrm{~h}^{-1}$ \\
& $3^{\circ} \mathrm{C}$ & $0.1^{\circ} \mathrm{C}$ \\
Lag and temp & $1 \mathrm{~h}$ & $1 \mathrm{~h}$ \\
& $1.5^{\circ} \mathrm{C}$ & $1{ }^{\circ} \mathrm{C}$ \\
\hline
\end{tabular}

by grid cell, across grouped grid cells (see below), and across all 8026 grid cells. When $R$ was calculated across multiple grid cells, the set of matched pairs for each grid cell was vertically concatenated to generate $\mathbf{M}$. This weighted each grid cell by its number of matched pairs for the calculation of $R$.

\section{c. Stratified matched pairs analysis}

We performed a series of stratified analyses by combining matched pairs that corresponded to various time periods and environmental conditions. For example, to differentiate between the effects of precipitation at different temperatures, we calculated $R$ for all matched pairs with equal temperature during the hour of precipitation using a sliding window spanning $1.5^{\circ} \mathrm{C}$. Using analogous methods, we assessed variability in $R$ across hours of the day, days of the week, and weeks of the year (Table 1). Finally, we performed an analysis that assessed variations in $R$ by precipitation rate and air temperature using a two-dimensional sliding window (Table 1).

\section{d. Lag analysis}

A lag analysis was performed for a more complete assessment of the effects of precipitation on motor vehicle crashes. We calculated $R$ (using the matched pairs technique) in the $0-48 \mathrm{~h}$ following the cessation of a precipitation event and matched this against periods a week earlier or before with no precipitation during the corresponding $0-48$-h stretch. We also performed an analysis that assessed variations in $R$ by lag and air temperature (Table 1).

\section{e. Confidence intervals and tests of significance}

A bootstrapping method was applied to the matched pairs to construct confidence intervals (Austin and Small 2014) for estimates of $R$. Specifically, 1000 matched pair bootstrap samples of size $N$ were drawn from the set of
$N$ matched pairs in $\mathbf{M}$. The $R$ for each bootstrap sample was calculated and the nonparametric percentile-based approach was used to calculate the $95 \%$ confidence intervals. In addition, we calculated a bootstrap $p$ value (Manly 2006) to assess whether $R_{g}$ was significantly different from $R_{A}$, where $R_{g}$ and $R_{A}$ are the relative accident risks calculated at grid cell $g$ and across all grid cells, respectively. Briefly, the $p$ values for individual grid cells were calculated by drawing 1000 matched pair bootstrap samples of size $n$, where $n$ is the number of matched pairs for $g$, from the matrix of all matched pairs, M. The $R$ for each bootstrap sample was calculated and the distribution was compared to $R_{g}$. Bootstrap distributions where $R_{g}$ was more extreme than the 99.5 th or 0.5 th percentile of the bootstrap sample were considered significant $(\alpha=0.01)$. For tests of significance, we discarded all grid cells with fewer than 10 matched pairs.

\section{Results}

A total of 85466 matched pairs were identified and they included 115900 motor vehicle crashes. The number of matched pairs varied considerably across grid cells due to the spatial variability in the number of crashes (Fig. 1). Overall, the RAR across all grid cells was 1.69 [1.66, 1.71]. Significant variation in RAR was observed across grid cells, with most grid cells along the interstates and highways greater than the RAR across all grid cells (Fig. 1).

\section{a. Variability in RAR by time}

RAR varied considerably by hour, day of the week, and season. Seasonally, maximum values were observed during the winter months and minimum values during the spring and fall (Fig. 2). Specifically, RAR peaks at $2.7[2.6,2.8]$ in late January and early February and reaches a nadir of $1.3[1.3,1.4]$ in early May and the end of October. There is a slight increase in RAR during the summer, culminating in a maximum of $1.5[1.5,1.6]$ at the end of August, and then a slow decline into fall (Fig. 2). RAR also varies by day of the week, with RAR greater during weekdays than during the weekend. In particular, RAR was greatest on Friday with an RAR of $1.8[1.7,1.8]$ and lowest during the weekend when RAR decreased to $1.6[1.5,1.6]$ for both Saturday and Sunday (Fig. 3). We also observed strong variation by hour of the day. For all days of the week, we observe RAR increasing during the morning with maximum values of $\sim 2$ in the late afternoon and minimum values of approximately 1.3 from 1200 to $0300 \mathrm{LT}$ (local time). However, this pattern varies slightly by day of the week. In particular, we observe large decreases in RAR during weekend nights and early mornings; and for some of the 

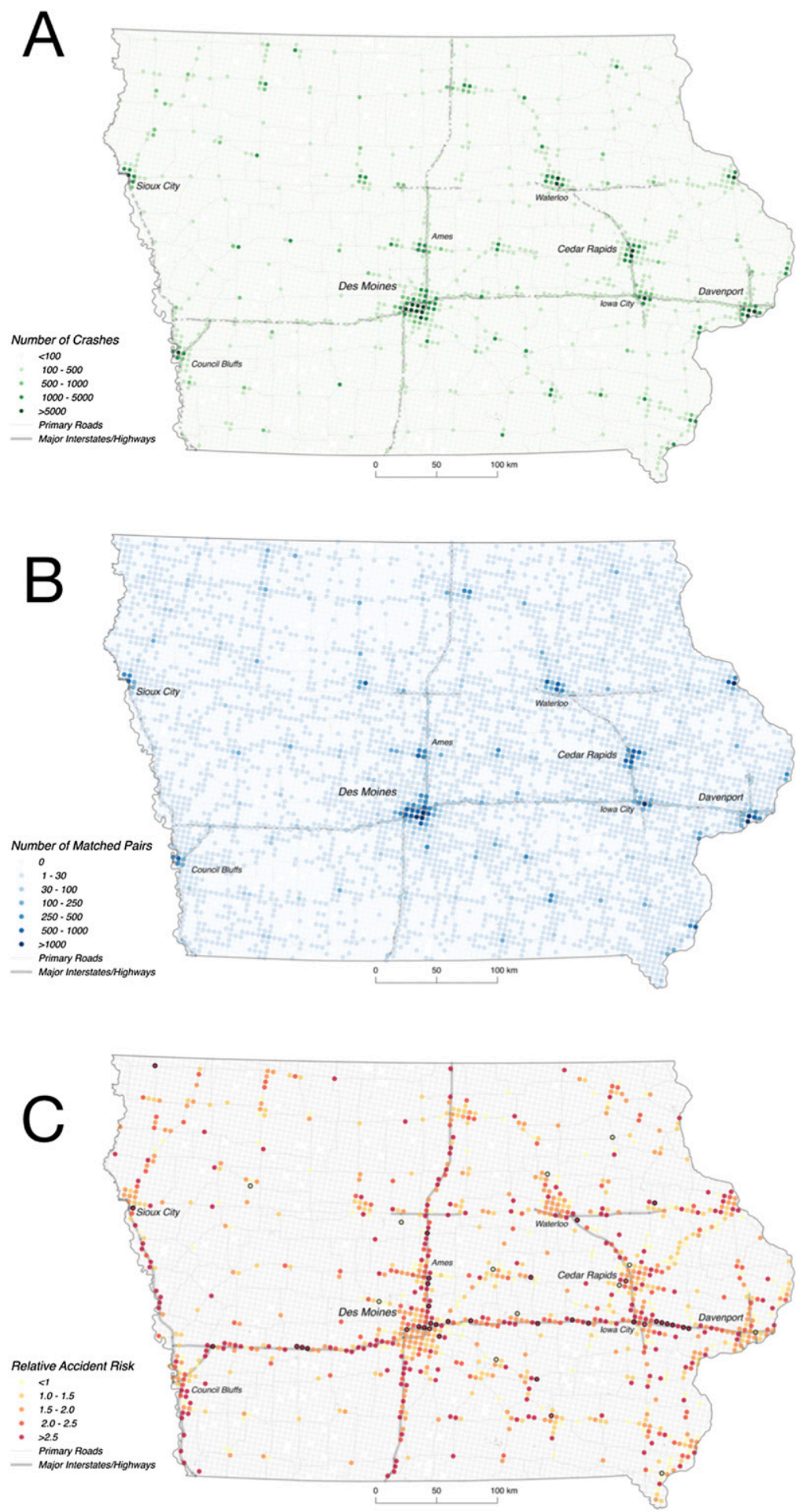

FIG. 1. (a) The total number of crashes per grid cell. The filled circles represent the grid cells. The major road networks are displayed in the background. (b) The total number of matched pairs per grid cell. Although the distribution of matched pairs closely matches the total number of crashes in (a), the number of matched pairs is significantly smaller as a result of the infrequency of hours with precipitation. (c) The RAR for each grid cell with $>30$ matched pairs. The dark outline around the filled circles indicate that the RAR is significantly different $(\alpha=0.01)$ than the RAR calculated across all grid cells (1.69). 

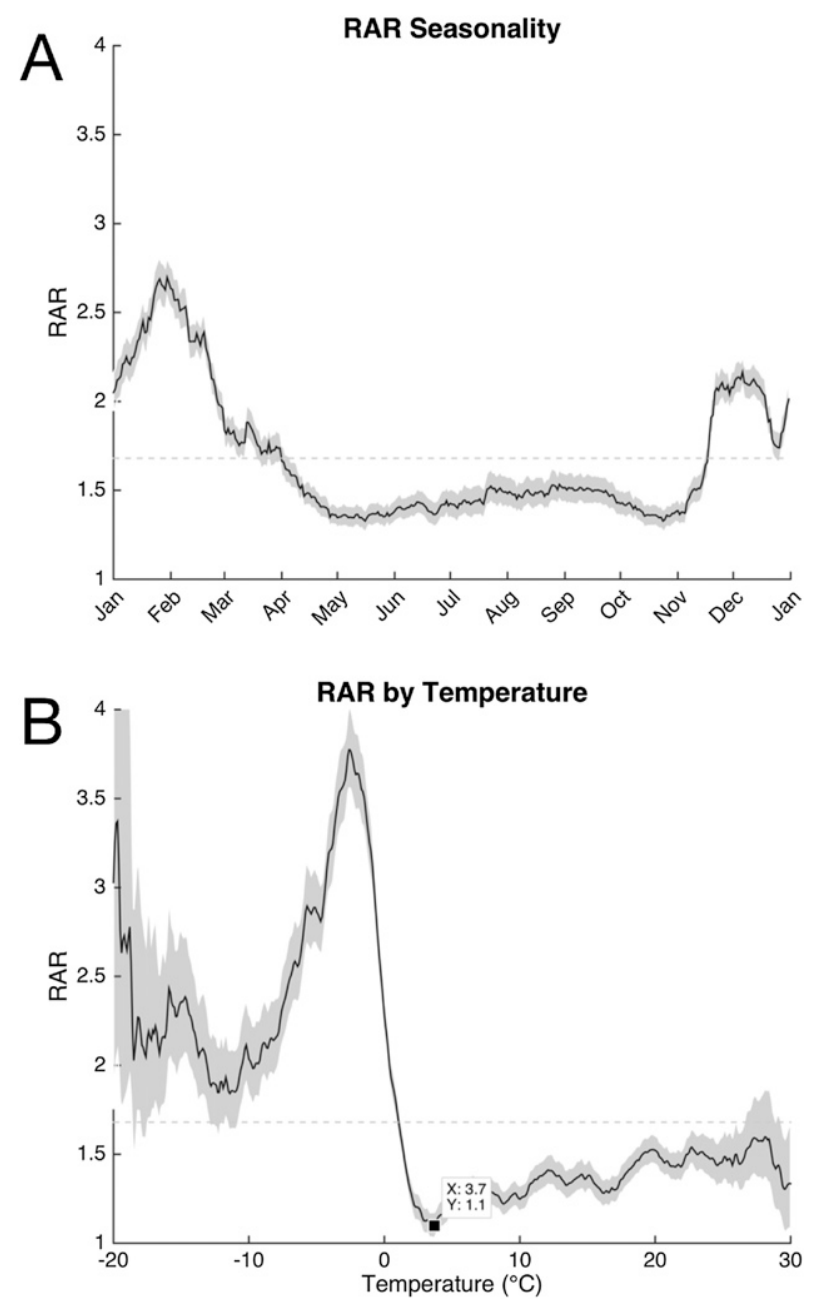

FIG. 2. (a) The average RAR across the year. The values are greatest in the winter and decrease in the summer. These differences are the result of changes in precipitation type. (b) The relationship between RAR and air temperature. The RAR increases to nearly 4 as the temperature decreases below $0^{\circ} \mathrm{C}$. There is a slight positive trend in RAR as temperatures increase from $3.7^{\circ} \mathrm{C}$. In both plots, the gray bands are $95 \%$ confidence intervals. For reference, the dotted horizontal line indicates the RAR calculated across all matched pairs.

weekdays, we observe maximums during midmorning and late afternoon hours (Fig. 3).

\section{b. RAR by temperature and precipitation rate}

RAR also varied strongly by temperature. RAR reached a minimum of $1.1[1.0,1.2]$ at $3.4^{\circ} \mathrm{C}$. As the air temperature decreased below $3.4^{\circ} \mathrm{C}$, RAR rapidly increased to $3.7[3.6,4.0]$ at $-2.5^{\circ} \mathrm{C}$ (Fig. 4). The RAR then decreased to $1.9[1.6,2.1]$ as temperatures reached $-11^{\circ} \mathrm{C}$. As temperatures increased from $3.4^{\circ} \mathrm{C}$, RAR gradually increased to approximately 1.6 as temperatures approached $28^{\circ} \mathrm{C}$. The relationship between RAR and precipitation rate is complex.
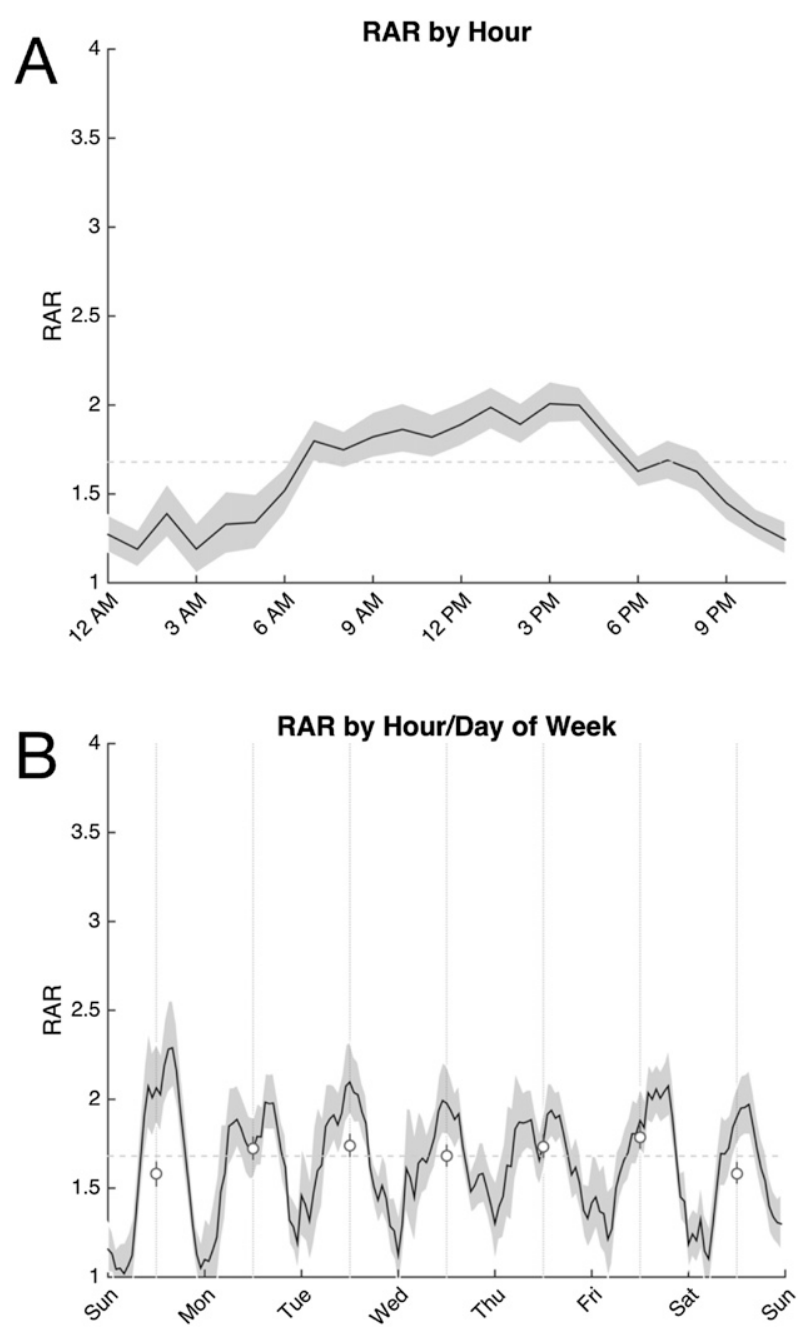

FIG. 3. (a) RAR by hour of the day. It shows that RAR varies significantly by hour of the day. A maximum RAR of 2 occurs near 1600 LT and a minimum of 1.2 occurs near 0300 LT. (b) RAR by hour and day of the week. It shows RAR varies across days of the week (circles with 95\% CI) with maximum RAR during the weekdays and minimum RAR during the weekend. It also shows the diurnal cycle in RAR varies by day [black line with shaded $95 \%$ contour interval (CI)]. For reference, the dotted horizontal line indicates the RAR calculated across all matched pairs and the vertical lines in (b) indicate the noon hour for each day.

There is a small increase in RAR near $1-2 \mathrm{~mm} \mathrm{~h}^{-1}$ and then a decrease to approximately $4 \mathrm{~mm} \mathrm{~h}^{-1}$. This small increase occurs only in frozen precipitation types (supplemental Fig. S1). RAR then slowly increases through approximately $22 \mathrm{~mm} \mathrm{~h}^{-1}$, but there are several significant decreases at 10,15 , and $20 \mathrm{~mm} \mathrm{~h}^{-1}$. When we examine the effects of RAR by temperature and precipitation rate (Fig. 4), we observe that precipitation coinciding with temperature $<0^{\circ} \mathrm{C}$ has an RAR $>2$ across all precipitation rates and is $>4$ for some rates near $0^{\circ} \mathrm{C}$, whereas $\mathrm{RAR}$ 


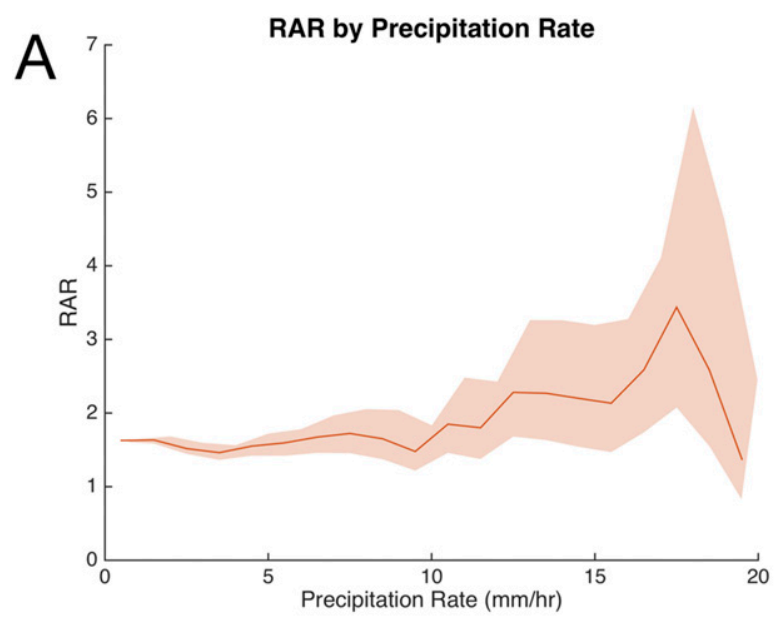

B
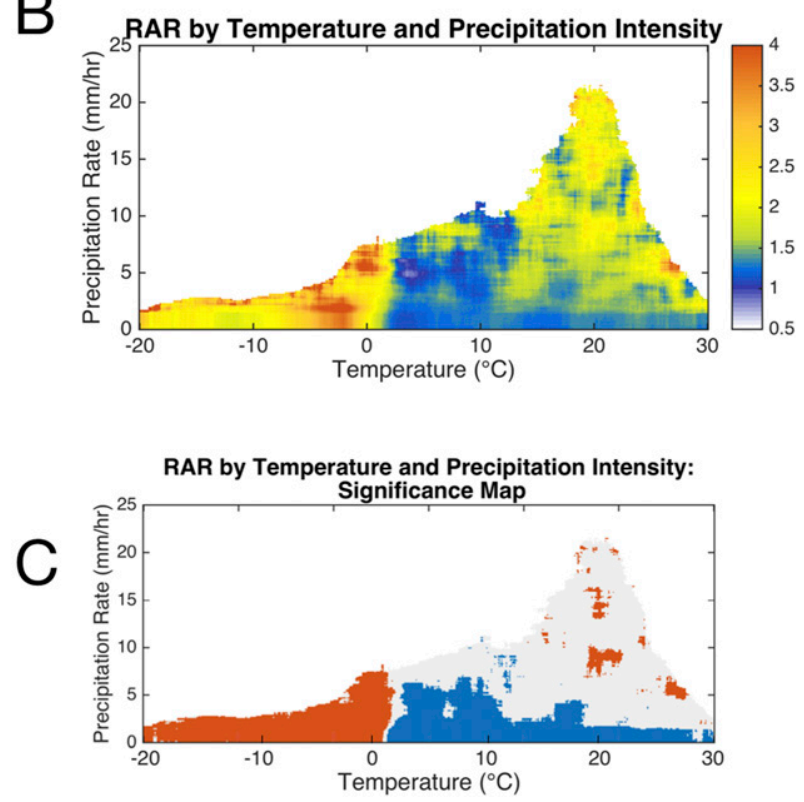

FIG. 4. (a) The relationship between RAR and the precipitation rate. There is a slight increasing trend in RAR with increasing temperature. For reference, the dotted horizontal line indicates the RAR calculated across all matched pairs. (b) RAR as a function of both temperature and precipitation intensity. (c) The areas in (b) that are significantly $(\alpha=0.01)$ above (red) and below (blue) the RAR across all matched pairs (1.69). Together these plots show that RAR is well above average at all precipitation rates when the temperatures is $<0^{\circ} \mathrm{C}$. RAR decreases dramatically as the temperature increases from $0^{\circ}$ to $10^{\circ} \mathrm{C}$ across nearly all precipitation rates. When the temperature is $>10^{\circ} \mathrm{C}$, light precipitation $\left(<5 \mathrm{~mm} \mathrm{~h}^{-1}\right)$ is associated with significantly low RAR, but RAR increases to $>2$ as rainfall rates increase $>10 \mathrm{~mm} \mathrm{~h}^{-1}$.

is relatively low for precipitation events where the temperature is $>0^{\circ} \mathrm{C}$. There appears to be an increase in RAR as the precipitation rate increases greater than $10 \mathrm{~mm} \mathrm{~h}^{-1}$ at temperatures $>15^{\circ} \mathrm{C}$. It should be noted that precipitation rates do not exceed $\sim 10 \mathrm{~mm} \mathrm{~h}^{-1}$ when temperatures are $<15^{\circ} \mathrm{C}$.

\section{c. RAR by lag}

When all matched pairs are included, the effect of precipitation on RAR is significant up to $20 \mathrm{~h}$ after precipitation ends (Fig. 5). However, the length of this lag effect varies by temperature with precipitation events that occur below $0^{\circ} \mathrm{C}$ corresponding to an $\mathrm{RAR}>1$ for up to $48 \mathrm{~h}$ after precipitation has ceased. On the other hand, lag effects are significant only up to $1 \mathrm{~h}$ for precipitation events that occur above $0^{\circ} \mathrm{C}$. In addition, there is some evidence that precipitation events above $0^{\circ} \mathrm{C}$ may be associated with an RAR $<1$ in the hours following the cessation of precipitation.

\section{Discussion}

The overall RAR during the study period was 1.69 ; that is, on average, precipitation increased the accident risk by nearly $70 \%$ in Iowa. This is consistent with estimates from previous studies (Keay and Simmonds 2006; Andrey et al. 2003; Andrey and Yagar 1993). However, RAR varied considerably across time, space, and environmental conditions. Specifically, our analysis shows that RAR ranged from 2.3 in January to 1.3 in May (Fig. 2). These extreme variations in RAR observed across seasons is a function of precipitation type, as Iowa weather is characterized by rain during the summer, and freezing rain and snow during the winter. This effect was highlighted by observations of variability in RAR by air temperature. Indeed, RAR increases from a minimum of 1.1 at $3.7^{\circ} \mathrm{C}$ and then rapidly increases to $>3$ at $-2.5^{\circ} \mathrm{C}$. This dramatic increase likely differentiates liquid and frozen precipitation types. Precipitation events with temperature just under $0^{\circ} \mathrm{C}$ are the most likely to be characterized by freezing rain, which significantly decreases friction with the road. Further, snow falling at these temperatures will have higher moisture content, which may reduce friction more than drier snow, which occurs at colder temperatures. The unusually low RAR near $3^{\circ} \mathrm{C}$ may be because the precipitation that occurs is primarily liquid at these temperatures, but drivers may be more cautious (e.g., reduction in speed), since it may not be clear if the rain is freezing on impact with the road.

RAR also gradually increases from 1.1 to 1.5 as temperatures increase from $3.7^{\circ}$ to $29^{\circ} \mathrm{C}$. This increase in RAR is likely due to greater precipitation rates at higher temperatures (Fig. 4). This finding is consistent with the results of previous studies (Hambly et al. 2013). However, we found that precipitation rate has a relatively complex relationship with RAR. These variations can be partially explained by relationships between rainfall rates and precipitation types (supplemental Fig. S1). For 

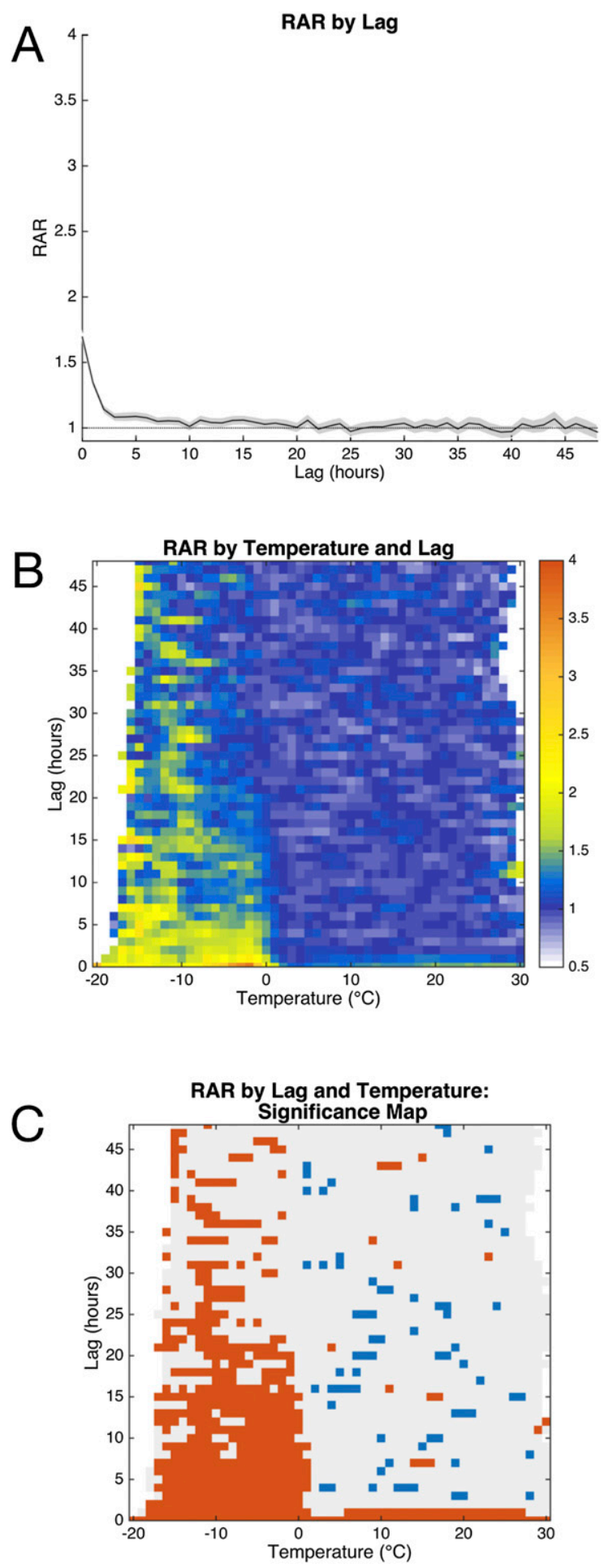

FIG. 5. (a) The lagged effect of precipitation on RAR. The plot shows that precipitation has a positive and significant effect for nearly $20 \mathrm{~h}$ following the cessation of a precipitation event. For reference, the dotted horizontal line indicates when RAR is equal to 0 (no precipitation effect). (b) The lag effect by air temperature. It shows that the lag effect can last for up to $48 \mathrm{~h}$ following precipitation events below $0^{\circ} \mathrm{C}$. On the other hand, rain events have only a 0-1-h lag effect before the RAR returns to 1 (and maybe below 1). (c) Where the RAR is significantly greater (red) or lower (blue) than 1 by temperature and lag. example, the increase near $2 \mathrm{~mm} \mathrm{~h}^{-1}$ is likely caused by the disproportionate number of matched pairs characterized by low precipitation rates during ice/snow events (supplemental Fig. S2). The local increases and decreases in the trend may have to do with associations between rainfall rate and storm type. For example, the decrease in RAR around $23-25 \mathrm{~mm} \mathrm{~h}^{-1}$ may be more frequent in short-term storms, which have been shown to have a smaller impact on RAR (Andrey and Yagar 1993). However, the small number of matched pairs above $10 \mathrm{~mm} \mathrm{~h}^{-1}$ make it difficult to determine the significance of the sinuous trends observed and these results should be treated with caution. A limitation of this analysis is that the estimates of precipitation rate are not precise, as we assumed that the hourly accumulations of precipitation were distributed evenly over the hour, whereas the rate of precipitation could have occurred over a short time period.

The average RAR varied significantly across grid cells (supplemental Fig. S3). An examination of RAR across grid cells suggests that interstates and major highways have, in general, slightly larger RAR values than smaller roads and highways (Fig. 1). This may be because of the higher speeds on these roads, which amplify the effect of precipitation on crashes (Jaroszweski and McNamara 2014).

RAR varies significantly by the hour of the day. RAR is at a minimum at midnight and then increases until it peaks at approximately $1500 \mathrm{LT}$. The pattern is broadly consistent for each day of the week (Fig. 3). However, many weekdays are characterized by a local maximum during the morning and the afternoon rush hour, with the afternoon rush hour significantly greater than the morning rush hour for the majority of the weekdays. The higher RAR corresponding to periods with higher traffic volume and density suggests an interaction effect between traffic volume and/or density and precipitation on RAR.

Although we primarily investigated the concurrent effect of precipitation on accident rates in this study, we also showed that a significant lag effect exists between precipitation and crashes (Fig. 5). The lag effect is strongly dependent on precipitation type with significant 1-h lags for liquid precipitation and significant lags up to $48 \mathrm{~h}$ for frozen precipitation events. Accordingly, the effect of frozen precipitation on crashes can be significantly underestimated if only concurrent effects of precipitation are assessed. The significant 1-h lag following liquid precipitation events conflicts with an earlier study that suggested that RAR immediately returned to 1 as rainfall ended (Andrey and Yagar 1993), and the findings suggest that the reduction in road friction is a significant contributor to an increase in crashes during rainfall events. The lag analysis also 
suggests that rainfall may have a slight lagged protective effect. Indeed, RAR is below 1 for a substantial portion of the hours following a liquid precipitation event (Fig. 5). This could be due to changes in driving behavior that take place during a precipitation event (e.g., slower speeds) that then carry over into the hours following the precipitation event. This explanation is similar to drivers becoming conditioned to adverse weather conditions if these conditions occur frequently over the course of a few days or months (Fridstrøm et al. 1995; Eisenberg and Warner 2005).

There were several limitations to this study. We did not include the effects of deicing chemicals that are added to roadways that decrease the freezing temperature. Thus, in some cases frozen precipitation may not have been accumulating despite temperatures below $0^{\circ} \mathrm{C}$. Future studies could employ data that indicate which roads are deiced to improve our understanding of the effects of various mitigation techniques on motor vehicle crashes. Further, although we performed a lag analysis to improve our understanding of the residual effects of precipitation, we did not account for the duration of a precipitation event, which has been shown to have an effect on crashes (Andrey and Yagar 1993). Also, although the matched pairs analysis accounts for typical traffic volume variability, it does not account for the effects of precipitation and precipitation type on traffic volume, which has been shown to decrease significantly during precipitation (Keay and Simmonds 2005; Doherty et al. 1993). Without traffic volume data, we cannot accurately describe the increased risk of an accident due to precipitation and the performed analysis generates conservative estimates of precipitation-related risks. Future studies could combine traffic data and weather data to make a more precise and accurate measure of precipitation effects on motor vehicle crashes. Finally, the 4-km resolution of the study allowed us to increase the number of crashes in each grid cell and calculate stable estimates of RAR across the state. However, the drawback to this approach is that the aggregation likely masked significant heterogeneity in RAR within a grid cell. Future studies can use more highly resolved radar data and perform the analysis at the scale of the road network to mitigate this limitation.

\section{Conclusions}

In this study we used weather radar and temperature data to assess the effects of precipitation and precipitation type on motor vehicle crashes. The data suggest that RAR varies considerably across time of day, day of week, and by season. The variation in RAR observed for time of day and day of week likely owe to an interaction effect between traffic volume/density and precipitation on crashes. We also observe a large variation in RAR by air temperature due to changes in precipitation type. The relationship between precipitation intensity and RAR is complex, partly due to associations between precipitation intensity and precipitation type but, in general, there is an increase in RAR as precipitation rates increase. We also showed that interstates and major highways are more sensitive to the effects of precipitation than smaller roads. This study can be used to inform future studies on the effects of weather on crashes. In particular, this study suggests that small changes in temperature and precipitation type can have large effects on crash risk, which is critical for understanding the future effects of climate change on crashes.

\section{REFERENCES}

Abdel-Aty, M., A.-A. Ekram, H. Huang, and K. Choi, 2011: A study on crashes related to visibility obstruction due to fog and smoke. Accid. Anal. Prev., 43, 1730-1737, doi:10.1016/j.aap.2011.04.003.

Andrey, J., and S. Yagar, 1993: A temporal analysis of rain-related crash risk. Accid. Anal. Prev., 25, 465-472, doi:10.1016/ 0001-4575(93)90076-9.

_ B. Mills, M. Leahy, and J. Suggett, 2003: Weather as a chronic hazard for road transportation in Canadian cities. Nat. Hazards, 28, 319-343, doi:10.1023/A:1022934225431.

Austin, P. C., and D. S. Small, 2014: The use of bootstrapping when using propensity-score matching without replacement: A simulation study. Stat. Med., 33, 4306-4319, doi:10.1002/ sim. 6276 .

Basagaña, X., J. P. Escalera-Antezana, P. Dadvand, Ò. Llatje, J. Barrera-Gómez, J. Cunillera, M. Medina-Ramon, and K. Pérez, 2015: High ambient temperatures and risk of motor vehicle crashes in Catalonia, Spain (2000-2011): A timeseries analysis. Environ. Health Perspect., 123, 1309-1316, doi:10.1289/ehp.1409223.

Doherty, S. T., J. C. Andrey, and J. C. Marquis, 1993: Driver adjustments to wet weather hazards. Climatol. Bull., 27, 154-164.

Edwards, J. B., 1999: The temporal distribution of road accidents in adverse weather. Meteor. Appl., 6, 59-68, doi:10.1017/ S1350482799001139.

Eisenberg, D., and K. E. Warner, 2005: Effects of snowfalls on motor vehicle collisions, injuries, and fatalities. Amer. J. Public Health, 95, 120-124, doi:10.2105/AJPH.2004.048926.

Fridstrøm, L., J. Ifver, S. Ingebrigtsen, R. Kulmala, and L. Krogsgård Thomsen, 1995: Measuring the contribution of randomness, exposure, weather, and daylight to the variation in road accident counts. Accid. Anal. Prev., 27, 1-20, doi:10.1016/ 0001-4575(94)E0023-E.

Hambly, D., J. Andrey, B. Mills, and C. Fletcher, 2013: Projected implications of climate change for road safety in Greater Vancouver, Canada. Climatic Change, 116, 613-629, doi:10.1007/ s10584-012-0499-0.

Jaroszweski, D., and T. McNamara, 2014: The influence of rainfall on road accidents in urban areas: A weather radar approach. Travel Behav. Soc., 1, 15-21, doi:10.1016/j.tbs.2013.10.005.

_ L. Chapman, and J. Petts, 2010: Assessing the potential impact of climate change on transportation: The need for an 
interdisciplinary approach. J. Transp. Geogr., 18, 331-335, doi:10.1016/j.jtrangeo.2009.07.005.

Jung, S., X. Qin, and D. A. Noyce, 2010: Rainfall effect on singlevehicle crash severities using polychotomous response models. Accid. Anal. Prev., 42, 213-224, doi:10.1016/j.aap.2009.07.020.

Keay, K., and I. Simmonds, 2005: The association of rainfall and other weather variables with road traffic volume in Melbourne, Australia. Accid. Anal. Prev., 37, 109-124, doi:10.1016/ j.aap.2004.07.005.

- and - 2006: Road accidents and rainfall in a large Australian city. Accid. Anal. Prev., 38, 445-454, doi:10.1016/ j.aap.2005.06.025.

Lin, Y., and K. E. Mitchell, 2005: The NCEP stage II/IV hourly precipitation analyses: Development and applications. 19th
Conf. on Hydrology, San Diego, CA, Amer. Meteor. Soc., 1.2. [Available online at https://ams.confex.com/ams/ Annual2005/techprogram/paper_83847.htm.]

Manly, B. F. J., 2006: Randomization, Bootstrap and Monte Carlo Methods in Biology. 3rd ed. Chapman \& Hall/CRC, $480 \mathrm{pp}$.

Qin, X., D. Noyce, C. Lee, and J. Kinar, 2007: Snowstorm event-based crash analysis. Transp. Res. Rec., 1948, 135-141, doi:10.3141/ 1948-15.

Qiu, L., and W. Nixon, 2008: Effects of adverse weather on traffic crashes: Systematic review and meta-analysis. Transp. Res. Rec., 2055, 139-146, doi:10.3141/2055-16.

U.S. DOT, 2011: How do weather events impact roads? [Available online at http://ops.fhwa.dot.gov/weather/q1roadimpact.htm.] 